\title{
Problemas de comportamiento y competencias psicosociales en niños y adolescentes institucionalizados*
}

\author{
Behavior Problems and Psychosocial Skills in \\ Institutionalized Children and Adolescents
}

Recibido: junio 15 de 2012 | Revisado: noviembre 30 de 2012 | Aceptado: diciembre 10 de 2012

\begin{abstract}
Martha P. Fernández-DaZA** ANTONIO FERNÁNDEZ-PARRA ***
\end{abstract}

Universidad de Granada, España

doi:10.11144/Javeriana.UPSY12-3.pccp

Para citar este artículo: Fernández-Daza, M. P. \& Fernández-Parra, A. (2013). Problemas de comportamiento y competencias psicosociales en niños y adolescentes institucionalizados. Universitas Psychologica, 12(3), 797-810. doi:10.11144/Javeriana. UPSY12-3.pccp

* Esta investigación ha sido financiada por el FO. NACIT (República Bolivariana de Venezuela) otorgada a la doctoranda Martha P. FernándezDaza. Ambos autores agradecen la colaboración prestada por el grupo HUM-129 del Plan Andaluz de Investigación y la Universidad de Granada.

** Doctoranda del Programa de Doctorado en Psicología Clínica y de la Salud de la Universidad de Granada. Becaria de posgrado del FONACIT (República Bolivariana de Venezuela). E-mail: mafercv@gmail.com

**** Profesor Titular de Universidad, en Psicopatología Infantil y Juvenil. Doctor en Psicología, Psicólogo Especialista en Psicología Clínica, Experto en Psicoterapia (EuroPsy). Director de la Clínica de Psicología de la Universidad de Granada, España Departamento de Personalidad, Evaluación y Tratamiento Psicológico. Facultad de Psicología. Campus Cartuja s/n. 18071 Granada, España. ResearcherID: H-9415-2013.E-mail: afparra@ugr.es
RESUMEN

El objetivo principal de este estudio es analizar los problemas de comportamiento de una muestra de preadolescentes y adolescentes venezolanos institucionalizados (acogimiento residencial), de edades comprendidas entre los 11 y 16 años. La muestra estuvo compuesta por 111 participantes institucionalizados en asociaciones civiles y entidades de protección del Estado y 111 controles igualados, provenientes de colegios tanto públicos como privados o subsidiados. Se evaluaron las habilidades psicosociales y académicas y los problemas de comportamiento internalizados y externalizados reportados por los informes de los padres, los cuidadores y los autoinformes mediante el Youth Self-Report (YSR) y la Child Behavior Checklist (CBCL). Los resultados del estudio muestran que los niños y adolescentes institucionalizados tienen menores competencias psicosociales y académicas, así como más problemas de comportamiento que los jóvenes que conviven con sus padres. Los padres y cuidadores apreciaron menores diferencias entre ambos grupos.

Palabras clave autores

Niños, adolescentes, problemas externalizantes, problemas internalizantes, competencias psicosociales, institucionalización, acogimiento residencial. Palabras clave descriptores

Psicología del desarrollo, Psicología educativa, investigación cuantitativa.

\section{A B S T R AC T}

The main objective of this study is analyze behavior problems of a sample of institutionalized (residential care) Venezuelan tweens and teens, ages between 11 and 16. The sample consisted of 111 participants institutionalized in civil associations and institutions state protection, and 111 matched controls from schools public and private or subsidized. The psychosocial and academic was assessed skills and internalizing and externalizing behavior problems reported by the reports of parents, carers and self reports. The Youth Self-Report (YSR) and Child Behavior Checklist (CBCL) were completed. The results of the study show that children and adolescents are less institutionalized psychosocial and academic skills, as well as, more externalized behavior problems and internalized self-reported that young people living with their parents. Parents, teachers and caregivers appreciated minor differences between the two groups. Findings obtained support the influence of early deprivation on psychosocial skills, emotional issues and behavioral in children and adolescents. It is considered that institutionalization is a risk factor for the harmonious development of young people. Key words authors

Children, Adolescents, Externalizing Problems, Internalizing Problems, Psychosocial Skills, Institutionalization, Residential Care.

Key words plus

Development Psychology, Educative Psychology, Quantitative Research. 


\section{Introducción}

Los niños conforman el grupo más vulnerable de un país y dentro de ese grupo destacan los niños privados del cuidado de sus progenitores, es decir los niños que no viven, al menos, con uno de sus progenitores por cualquier razón o circunstancia. En EE. UU., en el año 2006, había 510.000 niños en hogares de protección (Adoption and Foster Care Analysis and Reporting System, 2008). Según el Fondo de las Naciones Unidas para la Infancia-UNICEF (2009) más de 2.000.000 de niños están bajo tutela institucional en todo el mundo, de los cuales más de 800.000 viven en Europa Central y del Este y la Comunidad de Estados Independientes (ECE/CEI) y alrededor de 200.000 en América Latina y el Caribe. Sin embargo, debido a la falta de registro de muchas instituciones, seguramente esta cifra mundial dista mucho de la realidad.

El término institucionalización ha sido relacionado con diversas alternativas de actuación en materia de protección social infantil que, dependiendo del país, recibe otras denominaciones: acogimiento o cuidado residencial, cuidado institucional, colocación en entidad de atención, medida de abrigo, hogares de protección, entre otros. La institucionalización es una medida de protección que separa al niño de sus progenitores, de tal manera que permanecer en su casa no siga constituyendo un riesgo para su integridad (Benavides \& Miranda, 2007; Gómez, Muñoz \& Haz, 2007; Ison \& Morelato, 2008; Muñoz, Gómez \& Santamaría, 2008). Los niños institucionalizados son producto de la pobreza, el desarraigo, las familias disfuncionales, la orfandad, la carencia de lazos afectivos, el estrato social bajo, el maltrato, la enfermedad, la soledad, nexos familiares inmersos en la desprotección o el abandono.

En consecuencia, es el Estado y las instituciones privadas, muchas de ellas de tipo caritativo, los que asumen parte de este rol protector. En lo que respecta a los cuidadores de estas instituciones, no está claro si están formados para llevar a cabo el papel que se les asigna. Desde el punto de vista del desarrollo psicoafectivo, esta opción es cuestionable. Algunas investigaciones (Eapen, 2009; Grib- ble, 2007; Zeanah, Smyke, Koga \& Carlson, 2005) indican que las instituciones varían en cuanto a la calidad de la atención que proporcionan. Por lo general, los cuidadores tienen muchos niños a su cargo, lo que no permite una atención individualizada. En este sentido Smyke et al. (2007) observaron en su estudio que el comportamiento negativo de los menores estaba asociado a la poca calidad en los cuidados recibidos. Hay casos donde los derechos del niño se ven gravemente vulnerados y las condiciones para su desarrollo no son las mejores (Eapen, 2009; Gómez, Muñoz \& Santelices, 2008; Lieberknecht \& Dalbosco, 2007).

Estudios neurobiológicos sobre la atención institucional (Johnson, Browne \& Hamilton, 2006; Rutter, 2000; Rutter, O'Connor \& The English and Romanian Adoptees Study Team, 2004; Smyke et al., 2007; Smyke, Zeanah, Fox, Nelson \& Guthrie, 2010) sugieren que estos ambientes de alto estrés influyen en el desarrollo del cerebro y pueden causar deterioro cognitivo y déficit sociales. Los niños institucionalizados tienen una tasa mucho mayor de problemas psicosociales y de alto riesgo (Rutter, 2000) y muestran problemas tales como: agresividad, delincuencia, aislamiento o alteraciones de tipo emocional (Hetlinger, Simpkins \& Combs, 2000; Simsek, Erol, Öztop \& Münir, 2007). Según Gribble (2007) las privaciones físicas y emocionales de la institucionalización pueden dar lugar a un conjunto de problemas que incluyen dificultad en las relaciones afectivas e interpersonales, retrasos en el desarrollo físico y mental y problemas de lenguaje y de integración sensorial.

Por otra parte, la infancia institucionalizada también puede presentar trastornos de apego (Gómez et al., 2007; Johnson et al., 2006; Muris \& Maas, 2004; Smyke et al., 2007; Welsh, Viana, Petrill \& Mathias, 2007; Zeanah et al., 2005). Según dos series de metaanálisis realizados por Van den Dries, Juffer, van Ijzendoorn y Bakermans (2009), las experiencias de malos tratos, privación y abandono en los primeros meses de vida pueden tener consecuencias duraderas para la organización del apego. Sin embargo, cuando los niños son adoptados antes de los 12 meses de edad pueden superar la adversidad temprana y formar relaciones seguras 
con la misma frecuencia que sus homólogos normativos (Dozier \& Rutter, 2008).

Algunos autores (Ghera et al., 2009; Kreppner, O'Connor, Rutter \& The English and Romanian Adoptees Study Team, 2001) indican que el déficit de atención e hiperactividad puede constituir un síndrome de privación institucional. El estudio realizado en Chile por Méndez y González (2002) con menores institucionalizados mostró que el $37.9 \%$ de los identificados con problemas conductuales tenían un trastorno reactivo de la vinculación y que un $20.7 \%$ presentaban trastorno de apego.

Frente a toda esta literatura, Armsden, Pecora, Payne y Szatkiewicz (2000), evaluando a niños incluidos en programas de acogida (foster care) de EE. UU., concluyen que aunque un importante número de niños institucionalizados requiere de programas educativos y apoyo terapéutico, otros muchos están funcionando normalmente.

Los estudios publicados indican mayoritariamente que los menores institucionalizados presentan tasas más elevadas de problemas de comportamiento tanto de carácter externalizado como internalizado y mixtos (Armsden et al., 2000; Muris \& Maas, 2004; Rutter, 2000; Schmid, Goldbeck, Nutzel \& Fegert, 2008). Los comportamientos externalizados son aquellos que van dirigidos hacia los demás y causan molestias a otras personas, mientras que los de tipo internalizado incluyen sentimientos o estados que normalmente se consideran que están dirigidos hacia el propio individuo y le causan sufrimiento (Achenbach \& Edelbrock, 1983; Mash \& Graham, 2001). Comúnmente, los problemas que combinan ambas características no son agrupados o son considerados como mixtos. Así, los niños y adolescentes institucionalizados parecen presentar problemas internalizados (aislamiento social, alteraciones emocionales), externalizados (conductas desafiantes, agresivas y antisociales) y mixtos, de mayor gravedad que otros niños no institucionalizados.

Los problemas de comportamiento que presentan los niños y adolescentes institucionalizados son interpretados, habitualmente, desde una perspectiva transaccional (Sameroff \& Fiese, 2000) e incluso sistémico-conductual (Mash \& Graham,
2001). Se considera que la evolución del niño no puede desligarse del ambiente donde se desarrolla, es decir, sin analizar los efectos del medio sobre el niño. En este sentido, el ambiente institucional es considerado como un factor que, principalmente, altera el desarrollo psicológico infantil, afectando de manera significativa el comportamiento (Rutter, 2000).

En Venezuela el Instituto Autónomo Consejo Nacional de Derechos de Niños, Niñas y Adolescentes (IDENA) asume la protección integral de niños, niñas y adolescentes en situación de vulnerabilidad y riesgo social. Incluye a niños privados de su medio familiar bajo la tutela del Estado, los cuales son derivados a las Casas Comunales de Abrigo, las Unidades de Protección Integral y al Proyecto Familia Sustituta, dependiendo de las edades y características propias del caso. En el momento de este estudio, no se encontraron investigaciones publicadas que refieran la situación del niño institucionalizado en Venezuela, en centros o entidades de atención regidos por la actual legislación del país. No obstante, la UNICEF Venezuela (2012) señala que en el país hay 720 niños, niñas y adolescentes institucionalizados y 887 adolescentes privados de su libertad. Cabe la posibilidad de un subregistro debido a la falta de censo de algunas instituciones.

Los objetivos específicos planteados en el estudio fueron los siguientes: (1) identificar las competencias psicosociales y académicas (escolarización y rendimiento informado) de una muestra de adolescentes institucionalizados, en comparación con una muestra control no institucionalizada; (2) comparar ambos grupos respecto a los problemas de comportamiento autoinformados, así como los informados por sus tutores y/o cuidadores a través de los instrumentos utilizados en este estudio y (3) analizar la relación entre la información aportada por tutores y/o cuidadores con la autoinformada por los adolescentes. Se pretende comprobar si los niños y adolescentes venezolanos institucionalizados presentarán más problemas de comportamiento y menores competencias psicosociales que los niños no institucionalizados de su misma edad. Para la realización del estudio se adoptó un diseño ex post 
facto (también conocido como pseudoexperimental), de carácter transversal, con dos grupos de adolescentes seleccionados por estar institucionalizados o convivir con sus familias.

\section{Método}

\section{Participantes}

El universo de esta investigación estuvo constituido por 409 adolescentes y preadolescentes entre los 11 y 16 años del Distrito Federal Caracas y del Estado Miranda, específicamente de Baruta, Guarenas y Guatire, pertenecientes a colegios tanto públicos como privados o subsidiados. La muestra final quedó constituida por 222 participantes con una media de 13.3 años de edad ( $D E=1.24), 124$ mujeres y 98 varones, conformando dos grupos: los menores institucionalizados $(N=111)$ y el grupo control $(N=111)$. El grupo de adolescentes institucionalizados estaba constituido por 62 mujeres y 49 hombres (edad: $M=13.46 ; D E=1.41$ ), al igual que el grupo control con 62 mujeres y 49 hombres (edad: $M=13.14 ; D E=1.03$ ).

Los criterios de inclusión o exclusión fueron: estar o no institucionalizados en centros de protección de menores bajo la tutela del Estado o asociaciones civiles, tener entre 11 y 16 años de edad y estar escolarizados entre los cursos quinto de enseñanza primaria y noveno de bachillerato, ambos inclusive. Los motivos de ingreso de la muestra de adolescentes institucionalizados fueron: orfandad, haber sido niños de la calle o que la custodia a sus padres o familias hubiera sido retirada. La mayoría llevaban en la institución más de un año y habían ingresado entre los 8 y 11 años de edad. Las instituciones pertenecían a asociaciones civiles sin fines de lucro ( 2 instituciones) y entidades de protección del Estado (3 instituciones) que albergan a menores en situación de vulnerabilidad, excepto, menores infractores.

De las entidades que colaboraron en este estudio, tres eran de naturaleza religiosa y dos de ellas no tenían adscripción confesional. Las cinco instituciones trabajaban con menores entre los 0 y 18 años de edad. El grupo normalizado lo conformaban adolescentes de cuatro colegios de la comunidad que imparten educación normalizada. De los cuales, uno era privado y tres, subsidiados. Dos de los centros eran de naturaleza religiosa.

La muestra final de participantes institucionalizados se constituyó con todos aquellos niños y adolescentes que completaron la evaluación. El grupo control se formó como un grupo igualado en número, edad y sexo con el grupo institucionalizado, una vez se constituyó dicho grupo. Para esto se dividieron los niños y adolescentes no institucionalizados que fueron evaluados $(N=298)$ en tres niveles de edad (11-12, 13-14, y 15-16 años), y se seleccionaron al azar un número igual de niños y niñas a los que formaban parte del grupo institucionalizado en los mismos niveles de edad.

\section{Instrumentos}

Autoinforme del Comportamiento de Jóvenes (Youth Self-Report for Ages 11-18 [YSR]; Achenbach \& Rescorla, 2001)

Cuestionario reelaborado para evaluar la psicopatología de niños y adolescentes entre 11 y 18 años, a partir del informe del propio menor que aporta información sobre sus competencias psicosociales y problemas psicológicos. Está compuesto de dos partes: la primera de 17 ítems que hacen referencia a competencias psicosociales (habilidades deportivas, sociales y académicas); la segunda parte de 119 ítems: 14 describen conductas adaptativas o prosociales y el resto conductas desadaptativas. Los ítems están formulados en primera persona y se contestan en una escala tipo Likert con tres opciones: 0 (No es verdad), 1 (Algo verdad) y 2 (Muy cierto). La primera parte del cuestionario permite analizar los resultados de forma cuantitativa o cualitativa, considerando en este caso las respuestas como categorías.

El YSR/11-18 recoge información correspondiente a ocho escalas de conductas problema o síndromes de "banda estrecha" (retraimiento, quejas somáticas, ansiedad-depresión, problemas sociales, problemas de pensamiento, problemas de atención, conducta de ruptura de normas y conducta agresiva). Estas escalas se agrupan, a su vez, en un 
segundo nivel con dos patrones de carácter psicopatológico: los problemas internalizados o que causan malestar psicológico al sujeto y los problemas externalizados (conductas que causan malestar en el entorno del sujeto). Según Achenbach y Rescorla (2001) hay tres escalas de problemas internalizados (retraimiento, quejas somáticas, ansiedad-depresión) y dos de problemas externalizados (conducta de ruptura de normas y conducta agresiva).

La fiabilidad y validez del YSR ha sido ampliamente contrastada con escalas análogas de otros instrumentos (Abad, Forns, Amador \& Martorell, 2000; Achenbach \& Rescorla, 2001; Doyle, Mick $\&$ Biederman, 2007), en estudios transculturales (p. ej., Smokowski, Buchanan \& Bacallao, 2009), longitudinales (p. ej., Smokowski, Rose \& Bacallao 2009), realizados en Latinoamérica (Betancourt \& Andrade, 2008; Valencia \& Andrade, 2005) o con psicopatologías específicas (Zubeidat, FernándezParra, Sierra \& Salinas, 2007; Doyle et al., 2007). No existen datos específicos de fiabilidad y validez para la población estudiada en este trabajo.

\section{Cuestionario sobre el Comportamiento de}

Niños(as) de 6-18 años (Child Behavior CheckList [CBCL]; Achenbach \& Rescorla, 2001)

Es un instrumento dirigido a los padres, madres o tutores de menores entre los 6 y 18 años de edad que consta también de dos partes, como las descritas para el YSR. La valoración de la segunda parte permite igualmente realizar un perfil del comportamiento del niño, a través de ocho escalas o síndromes de "banda estrecha": aislamiento, síntomas somáticos, ansiedad/depresión, problemas sociales, problemas de pensamiento, problemas de atención, conducta de ruptura de normas y conducta agresiva. Esas escalas se agrupan en otras dos escalas de "banda ancha”, síndromes internalizados y síndromes externalizados, así como una puntuación total del cuestionario que incluye otros ítems no considerados en ninguna de las escalas anteriores.

La fiabilidad test-retest de esta escala, con un intervalo de una semana, es de 0.99 para la parte de adaptación social y rendimiento académico y de 0.95 para la parte del listado de conductas. La consistencia interna de la escala ha quedado demostrada en varias ocasiones (Achenbach \& Edelbrock, 1983; Caprara \& Pastorelli, 1989). Adicionalmente, al tratarse de una de las escalas más cuidadosamente validadas, es preferentemente utilizada como instrumento de screening en el ámbito de la psicopatología infantil (Luoma et al., 2004). Este instrumento ha sido utilizado en numerosos estudios transculturales (Rescorla et al., 2007), con escalas análogas de otros instrumentos (Achenbach et al., 2008; Hudziak, Copeland, Stanger \& Wadsworth, 2004) o con población vulnerable (Armsden et al., 2000). El CBCL ha sido aplicado a niños venezolanos con edades entre 5 y 12 años (Montiel, Montiel \& Peña, 2007), aunque no existen datos específicos para la población aquí estudiada.

\section{Procedimiento}

Antes de la aplicación de los instrumentos, se utilizó el juicio de cuatro expertos venezolanos en el campo de la Psicología, a los cuales se les entregaron las versiones oficiales en español de los dos instrumentos, el YSR y el CBCL, para que desde el punto de vista de contenido y forma determinaran la adecuada comprensión de cada uno de los ítems que conforman los instrumentos, con el objeto de evitar ambigüedades en los ítems formulados para niños y adolescentes venezolanos.

Después se procedió a seleccionar instituciones educativas y de protección de menores para obtener la muestra de niños, niñas y adolescentes definida por los criterios de inclusión. Posteriormente, se contactó con cada una de las instituciones a fin de informarles sobre el estudio a realizar. Una vez obtenida la autorización de las instituciones interesadas en formar parte de la investigación, se seleccionaron los participantes, y se procedió a obtener la autorización individual para la recogida de datos de los padres o tutores, en el caso de niños de centros educativos. Así, se remitió a los padres un documento de consentimiento informado y una hoja informativa sobre la investigación que se iba a llevar a cabo. Para los niños institucionalizados no fue necesaria la autorización individual de los padres, porque la custodia estaba a cargo de las entidades correspondientes. 
En el caso de los niños y adolescentes no institucionalizados se invitó a participar en el estudio a todos los integrantes de los cursos quinto de enseñanza primaria y noveno de bachillerato, ambos inclusive, que cumplían los criterios de edad. Solo se trabajó con aquellos con los que se obtuvo la autorización de sus padres o representantes legales. Respecto a los adolescentes institucionalizados, se contó con todos los que cumplían los criterios de inclusión y que no estuvieron enfermos o faltaron por algún motivo a alguna de las sesiones de evaluación. En todos los casos la institución había dado previamente su consentimiento. Durante la evaluación se informó a todos los participantes que se estaba realizando un estudio para el que se requería su participación que ya había sido autorizada por sus padres o representantes legales. Se les indicó, asimismo, que su participación era voluntaria.

La aplicación del instrumento YSR se realizó en dos sesiones de 30 minutos cada una y de forma grupal. A los niños institucionalizados se les ad- ministró en su tiempo libre, en espacios cómodos y tranquilos, y al grupo control durante su jornada escolar en su salón de clases y, generalmente, en presencia de algún docente.

Durante la evaluación se produjeron algunos problemas de comprensión de los ítems, que fueron solventados por los evaluadores. Al finalizar, se le entregó a cada niño de las instituciones escolares el CBCL para que lo llevara a casa y fuera respondido por sus padres. En el caso de los niños institucionalizados, se les entregó a sus cuidadores principales o habituales con los que compartían las actividades diarias extraescolares y eran responsables de su cuidado.

\section{Resultados}

Ya que la distribución por sexo de ambos grupos fue idéntica, se analizaron las posibles diferencias respecto a la edad, sin que se encontraran diferencias estadísticamente significativas entre los grupos

TABLA 1

Competencias psicosociales informadas mediante el YSR y CBCL

\begin{tabular}{|c|c|c|c|c|c|c|}
\hline \multirow[b]{3}{*}{ Escalas } & \multicolumn{4}{|c|}{ Grupos } & \multirow[b]{3}{*}{$t$} & \multirow[b]{3}{*}{$p$} \\
\hline & \multicolumn{2}{|c|}{$\begin{array}{l}\text { Institucionalizado } \\
\quad(N=111)\end{array}$} & \multicolumn{2}{|c|}{$\begin{array}{c}\text { Control } \\
(N=111)\end{array}$} & & \\
\hline & $M$ & $\mathrm{DE}$ & $M$ & $\mathrm{DE}$ & & \\
\hline YSR: Participación en deportes & 2.15 & 0.974 & 1.82 & 1.138 & 2.345 & $0.02 *$ \\
\hline YSR: Nivel de actividad en pasatiempos & 1.91 & 1.149 & 1.37 & 1.152 & 3.502 & $0.001 *$ \\
\hline YSR: Grupos a los que pertenece & 0.43 & 0.838 & 0.5 & 0.808 & -0.571 & 0.569 \\
\hline YSR: Grado de implicación en grupos & 0.75 & 1.203 & 0.97 & 1.225 & -1.343 & 0.181 \\
\hline YSR: Trabajos-tareas que realiza & 1.75 & 1.066 & 1.09 & 1.092 & 4.542 & $0.0001^{*}$ \\
\hline YSR: Habilidad para llevar a cabo tareas & 1.95 & 0.866 & 1.48 & 1.189 & 3.33 & $0.001 *$ \\
\hline YSR: Amigos(as) íntimos & 2.24 & 0.63 & 2.24 & 0.744 & -0.058 & 0.953 \\
\hline YSR: Rendimiento escolar & 1.76 & 0.801 & 2.04 & 0.84 & -2.53 & $0.012 *$ \\
\hline CBCL: Participación en deportes & 1.45 & 1.093 & 1.79 & 1.005 & -2.37 & $0.019 *$ \\
\hline CBCL: Nivel de actividad en pasatiempos & 1.2 & 1.124 & 1.56 & 1.017 & -2.448 & $0.015^{*}$ \\
\hline CBCL: Grupos a los que pertenece & 0.06 & 0.279 & 0.27 & 0.65 & -3.054 & $0.003 *$ \\
\hline CBCL: Grado de implicación en grupos & 0.16 & 0.654 & 0.44 & 0.96 & -2.548 & $0.012 *$ \\
\hline CBCL: Trabajos-tareas que realiza & 1.61 & 1.259 & 1.59 & 1.005 & 0.130 & 0.897 \\
\hline CBCL: Habilidad para llevar a cabo tareas & 1.53 & 1.125 & 1.76 & 0.917 & -1.627 & 0.105 \\
\hline CBCL: Amigos(as) íntimos & 2.1 & 0.369 & 2.1 & 0.537 & -0.073 & 0.942 \\
\hline CBCL: Rendimiento escolar & 1.91 & 2.353 & 2.03 & 0.765 & -0.5 & 0.618 \\
\hline
\end{tabular}

$* p<0.05$

Fuente: elaboración propia. 
$(t(220)=1.954, p=0.052$, n. s. $)$. Sí se encontraron diferencias en relación al nivel de escolarización, ya que 38 (34.2\%) adolescentes del grupo institucionalizado cursaban estudios primarios y 73 (65.8\%) estudios secundarios, mientras que en el grupo control 18 participantes (16.2 \%) cursaban estudios primarios y 93 (83.8 \%) estudios secundarios. El análisis estadístico reveló que estas diferencias en la distribución de los participantes de ambos grupos eran significativas $\left(\chi^{2}(1)=9.552, p=0.002\right)$.

En la Tabla 1 se muestran los resultados obtenidos sobre las características de los participantes de ambos grupos respecto a competencias psicosociales valoradas cuantitativamente, tal y como informaron ellos mismos a través del YSR o los padres y cuidadores a través del CBCL. Se encontraron diferencias significativas entre ambos grupos respecto a varias de las variables medidas a través del YSR, en las que los niños del grupo institucionalizado informaban de una mejor y mayor ejecución que los niños del grupo control, excepto, en rendimiento escolar. En lo que respecta a las competencias psicosociales reportadas a través del CBCL por padres y cuidadores, también se descubrieron diferencias significativas en algunas variables, siendo los niños institucionalizados quienes tienen puntajes más bajos en comparación con el grupo control.

Los resultados de las características psicosociales que fueron analizadas como variables cualitativas ordinales se presentan en la Tabla 2. Se puede observar que solo hubo diferencias significativas en las informadas mediante el CBCL por padres y cuidadores, en las que los niños institucionalizados muestran peores competencias.

Respecto a los problemas de comportamiento evaluados mediante autoinforme a través del YSR

TABLA 2

Características psicosociales informadas mediante la YSR y el CBCL

\begin{tabular}{|c|c|c|c|c|}
\hline \multirow[b]{2}{*}{ Variables } & \multicolumn{2}{|c|}{ Grupos } & \multirow[b]{2}{*}{$\chi^{2}$} & \multirow[b]{2}{*}{$p$} \\
\hline & $\begin{array}{l}\text { Institucionalizado } \\
\quad(N=111)\end{array}$ & $\begin{array}{c}\text { Control } \\
(N=111)\end{array}$ & & \\
\hline $\begin{array}{l}\text { YSR-Amigos(as) íntimos } \\
\text { Ninguno } \\
1 \\
2 \text { o } 3 \\
4 \text { o más }\end{array}$ & $\begin{array}{l}13 \\
25 \\
32 \\
41\end{array}$ & $\begin{array}{l}21 \\
20 \\
28 \\
42 \\
\end{array}$ & 2.717 & 0.437 \\
\hline $\begin{array}{l}\text { YSR-Veces a la semana que comparte con amigos } \\
\text { Nunca } \\
1 \text { o } 2 \\
3 \text { o más }\end{array}$ & $\begin{array}{l}40 \\
21 \\
50 \\
\end{array}$ & $\begin{array}{l}30 \\
32 \\
49 \\
\end{array}$ & 3.722 & 0.156 \\
\hline $\begin{array}{l}\text { YSR-Juega y hace sus tareas solo } \\
\text { Peor que los demás } \\
\text { Igual que los demás } \\
\text { Mejor que los demás }\end{array}$ & $\begin{array}{l}21 \\
46 \\
44 \\
\end{array}$ & $\begin{array}{c}15 \\
44 \\
52\end{array}$ & 1.711 & 0.425 \\
\hline $\begin{array}{l}\text { CBCL-Amigos(as) íntimos } \\
\text { Ninguno } \\
1 \\
2 \text { o } 3 \\
4 \text { o más }\end{array}$ & $\begin{array}{l}17 \\
20 \\
55 \\
19 \\
\end{array}$ & $\begin{array}{l}18 \\
23 \\
33 \\
34\end{array}$ & 9.944 & $0.019 *$ \\
\hline $\begin{array}{l}\text { CBCL-Veces a la semana que comparte con amigos } \\
\text { Nunca } \\
1 \text { o } 2 \\
3 \text { o más }\end{array}$ & $\begin{array}{l}51 \\
47 \\
13 \\
\end{array}$ & $\begin{array}{l}37 \\
34 \\
37 \\
\end{array}$ & 15.796 & $0.0001 *$ \\
\hline $\begin{array}{l}\text { CBCL-Juega y hace sus tareas solo } \\
\text { Peor que los demás } \\
\text { Igual que los demás } \\
\text { Mejor que los demás }\end{array}$ & $\begin{array}{l}16 \\
85 \\
10\end{array}$ & $\begin{array}{c}7 \\
80 \\
21\end{array}$ & 7.537 & $0.023 *$ \\
\hline
\end{tabular}

Nota. La tabla muestra el número de sujetos que presenta cada característica.

$* p<0.05$

Fuente: elaboración propia. 
(Tabla 3), se hallaron diferencias estadísticamente significativas entre ambos grupos en todas las escalas, siendo los niños institucionalizados los que obtuvieron puntuaciones superiores en comparación con el grupo control. Esto no fue así cuando se consideraron los informes de los padres o cuidadores a través del CBCL.

Por último, la Tabla 4 presenta las correlaciones entre informantes respecto a los problemas de comportamiento evaluados. Los informantes fueron los propios adolescentes y los padres o cuidadores. Las correlaciones más altas entre informantes correspondieron a los problemas de comportamiento externalizado (escalas de ruptura de normas y conducta agresiva, y suma total o síndrome externalizado), además a los problemas de atención tanto para la muestra total como para los dos grupos por separado. Considerando la muestra total, los informes de padres y cuidadores correlacionaban significativamente con los autoinfor- mes de los adolescentes, en todas las escalas. Cuando se consideraron los grupos por separado se observó que también hubo una correlación significativa en todas las escalas en la información aportada por adultos y adolescentes del grupo institucionalizado. Sin embargo, los informes del grupo control correlacionaron significativamente en todas las escalas, excepto, en las escalas de aislamiento depresivo, problemas del pensamiento y conductas agresivas.

\section{Discusión}

El objetivo general del presente estudio fue analizar los problemas de comportamiento autoinformados por jóvenes y reportados por padres o cuidadores, a través de dos instrumentos, YSR y CBCL, en una muestra de niños y adolescentes venezolanos seleccionados con base en el hecho de estar insti-

TABLA 3

Problemas de comportamiento de niños y niñas informados mediante la YSR y el CBCL

\begin{tabular}{|c|c|c|c|c|c|c|}
\hline \multirow[b]{3}{*}{ Escalas } & \multicolumn{4}{|c|}{ Grupos } & \multirow[b]{3}{*}{$t$} & \multirow[b]{3}{*}{$p$} \\
\hline & \multicolumn{2}{|c|}{$\begin{array}{l}\text { Institucionalizado } \\
\quad(N=111)\end{array}$} & \multicolumn{2}{|c|}{$\begin{array}{l}\text { Control } \\
(\mathrm{N}=111)\end{array}$} & & \\
\hline & $M$ & $D E$ & $M$ & $D E$ & & \\
\hline YSR: Ansiedad/Depresión & 6.55 & 4.602 & 3.89 & 4.391 & 4.402 & $0.0001 *$ \\
\hline YSR: Síntomas depresivos & 5.23 & 3.479 & 3.82 & 2.643 & 3.389 & $0.001 *$ \\
\hline YSR: Quejas Somáticas & 6.33 & 4.348 & 4.38 & 3.838 & 3.534 & $0.0001^{*}$ \\
\hline YSR: Problemas Sociales & 5.88 & 3.972 & 3.98 & 3.4 & 3.83 & $0.0001^{*}$ \\
\hline YSR: Problemas Pensamiento & 8.69 & 4.518 & 6.95 & 3.951 & 3.052 & $0.003 *$ \\
\hline YSR: Problemas de Atención & 7.45 & 3.992 & 4.87 & 3.371 & 5.194 & $0.0001^{*}$ \\
\hline YSR: Ruptura de Normas & 8.46 & 4.624 & 6.35 & 4.263 & 3.532 & $0.001 *$ \\
\hline YSR: Conductas Agresivas & 10.59 & 6.267 & 7.65 & 5.516 & 3.706 & $0.0001 *$ \\
\hline YSR: Síndromes Internalizados & 18.12 & 10.754 & 12.09 & 9.127 & 4.494 & $0.0001 *$ \\
\hline YSR: Síndromes Externalizados & 18.97 & 10.025 & 14 & 9.2 & 3.842 & $0.0001^{*}$ \\
\hline CBCL: Ansiedad/Depresión & 3.48 & 3.408 & 3.46 & 2.908 & 0.034 & 0.973 \\
\hline CBCL: Síntomas depresivos & 2.57 & 2.429 & 2.70 & 2.285 & -0.427 & 0.67 \\
\hline CBCL: Quejas Somáticas & 2.48 & 2.327 & 2.26 & 2.03 & 0.739 & 0.461 \\
\hline CBCL: Problemas Sociales & 2.99 & 4.022 & 3.48 & 2.975 & -1.024 & 0.307 \\
\hline CBCL: Problemas Pensamiento & 1.61 & 2.701 & 1.09 & 1.483 & 1.75 & 0.081 \\
\hline CBCL: Problemas de Atención & 4.74 & 4.867 & 4.52 & 3.384 & 0.388 & 0.699 \\
\hline CBCL Ruptura de Normas & 4.01 & 4.383 & 3.88 & 4.067 & 0.226 & 0.821 \\
\hline CBCL: Conductas Agresivas & 7.05 & 7.834 & 7.26 & 5.875 & -0.219 & 0.827 \\
\hline CBCL: Síndromes Internalizados & 8.52 & 6.554 & 8.43 & 5.646 & 0.117 & 0.907 \\
\hline CBCL: Síndromes Externalizados & 11.06 & 11.809 & 11.14 & 9.543 & -0.052 & 0.958 \\
\hline
\end{tabular}

$* p<0.05$

Fuente: elaboración propia. 


\section{TABLA 4}

Correlaciones entre los informes de los adolescentes y los de padres/cuidadores respecto a los problemas de comportamiento del niño

\begin{tabular}{lccc}
\hline \multicolumn{1}{c}{ Escalas } & $\begin{array}{c}\text { Muestra Total } \\
(\mathrm{N}=222)\end{array}$ & $\begin{array}{c}\text { Grupo Institucionalizado } \\
(\mathrm{N}=111)\end{array}$ & $\begin{array}{c}\text { Grupo Control } \\
(\mathrm{N}=111)\end{array}$ \\
\hline I. Ansiedad/Depresión & $0.229^{* *}$ & $0.2^{*}$ & $0.285^{* *}$ \\
II. Síntomas depresivos & $0.16^{*}$ & $0.216^{*}$ & 0.109 \\
III. Quejas Somáticas & $0.232^{* *}$ & $0.222^{*}$ & $0.237^{*}$ \\
IV. Problemas Sociales & $0.234^{* *}$ & $0.238^{*}$ & $0.296^{* *}$ \\
V. Problemas Pensamiento & $0.247^{* *}$ & $0.308^{* *}$ & 0.084 \\
VI. Problemas de Atención & $0.378^{* *}$ & $0.464^{* *}$ & $0.266^{* *}$ \\
VII. Ruptura de Normas & $0.451^{* *}$ & $0.536^{* *}$ & $0.366^{* *}$ \\
VIII. Conductas Agresivas & $0.275^{* *}$ & $0.429^{* *}$ & 0.095 \\
Síndromes Internalizados & $0.242^{* *}$ & $0.284^{* *}$ & $0.209^{*}$ \\
Síndromes Externalizados & $0.35^{* *}$ & $0.504^{* *}$ & $0.196^{*}$ \\
\hline
\end{tabular}

$* p<0.05 ; * *<<0.01$.

Fuente: elaboración propia.

tucionalizados o convivir con sus familias. Así, se planteó en primer lugar identificar las competencias psicosociales y académicas de niños y adolescentes institucionalizados en comparación con una muestra no institucionalizada. La revisión bibliográfica permite afirmar que las privaciones que han vivido y a las que están expuestos los niños institucionalizados pueden conllevar a una serie de alteraciones, no solo a nivel físico sino también académico y socioemocional, que afectan su funcionamiento. Los resultados obtenidos muestran que el rendimiento escolar de los niños institucionalizados fue significativamente inferior que el de los niños que conviven con sus padres, aunque solo cuando informaban los propios menores. Igualmente, los niños institucionalizados estaban escolarizados en cursos inferiores a los que les correspondían a su edad, en los que sí estaban escolarizados los participantes del grupo control. Este dato parece corroborar los informes de los propios adolescentes. El hecho de que la información aportada por padres/cuidadores no refleje esta misma situación puede ser debido al menor desconocimiento de los cuidadores sobre la situación académica de los niños institucionalizados, ya que no son responsables de este ámbito de sus vidas.

Los resultados sobre el rendimiento escolar se relacionan con los hallazgos encontrados por Behen, Helder, Rothermel, Solomon y Chugani (2008) donde niños que habían experimentado graves carencias mostraron déficits a nivel funcional a pesar de la integridad de las funciones básicas. En este caso, las carencias asociadas a la institucionalización y condiciones previas que llevan a este tipo de acogimiento, parecen influir en el rendimiento de los menores.

Sin embargo, los niños en acogimiento residencial afirmaban involucrarse más en deportes, pasatiempos y en los trabajos o tareas de la casa o comunidad, así como tener una mejor habilidad para llevarlas a cabo en comparación con los otros adolescentes. Esto podría deberse a que en las diferentes instituciones se distribuyen las responsabilidades para llevar a cabo el mantenimiento de la casa entre todos los integrantes, y que los menores acogidos tienen más posibilidades de participar en este tipo de actividades extraescolares. Sin embargo, estos resultados no fueron corroborados por los informes de padres y cuidadores a través del CBCL, ya que los niños institucionalizados obtuvieron puntajes inferiores en casi todas las competencias psicosociales evaluadas con respecto al grupo de niños que conviven con su familia. Según los informes de padres y cuidadores también hubo diferencias significativas en relación con las veces por semana que los menores comparten su tiempo con amigos, juegan y hacen sus tareas solos, o en el número de amigos íntimos. Se observa con estos resultados que las redes de soporte social con que cuenta el niño 
institucionalizado son menores, tal y como señalan Eapen (2009) y Rutter (2000).

No parece existir una explicación clara sobre estos resultados diferenciales obtenidos cuando se comparan los autoinformes o las valoraciones de padres/cuidadores. Podría pensarse que los adultos en general valoran en menor medida este tipo de actividades, y son menos sensibles a diferencias en la manera como niños y adolescentes se involucran en estas.

En lo que respecta a la comparación de ambos grupos respecto a los problemas de comportamiento externalizados, internalizados y mixtos autoinformados, se puede afirmar la presencia de diferencias tanto en las escalas de banda estrecha (problemas específicos) como en la escalas de banda amplia (síndromes o puntuaciones totales). En los autoinformes se hallaron diferencias significativas en todas las escalas de síndromes de banda estrecha de todo tipo, siendo los niños institucionalizados los que alcanzaron puntuaciones peores en comparación con el grupo control. No obstante, en los informes de los padres y cuidadores no se encontraron diferencias significativas entre ambos grupos.

Estos resultados difieren parcialmente de los hallazgos de Smyke et al. (2007), ya que los autores encontraron que los problemas externalizados e internalizados no fueron más comunes en los niños institucionalizados que los niños que viven con sus familias. Sin embargo, los resultados sí se asemejan a los obtenidos en investigaciones previas realizadas con población anglosajona (Armsden et al., 2000; Muris \& Maas; 2004; Rutter, 2000; Schmid et al., 2008). Los resultados del presente estudio indican, en cualquier caso, que los adolescentes institucionalizados presentan índices más elevados de problemas de comportamiento que los que viven con sus familias, cuando estos son autoinformados, pero también indican que esta información no se ve confirmada por los adultos responsables de su cuidado.

Precisamente, las diferencias encontradas a lo largo del estudio entre ambos tipos de informantes, los propios menores y sus padres o cuidadores, llevaron a analizar la relación entre la información que cada uno de ellos aportaba acerca de los problemas de comportamiento. En general las correlaciones más altas entre ambos tipos de informantes correspondieron a los problemas de comportamiento externalizado, hallazgos que concuerdan con los resultados del estudio de Reijneveld, Brugman, Verhulst y Verloove (2005). Igualmente, existe una correlación alta con los problemas de atención, tanto para el total de la muestra como para cada uno de los grupos del estudio. Sin embargo, el acuerdo entre informantes disminuye respecto a los problemas internalizados, que son de tipo emocional y que afectan al propio individuo pero que son menos evidentes para los adultos. Es más, en el grupo control la correlación fue muy baja y no significativa para las escalas de síntomas depresivos, problemas de pensamiento e incluso conductas agresivas. Pese a todo, considerando el total de la muestra, los informes de padres y cuidadores correlacionaban significativamente con los autoinformes de los adolescentes en todas las escalas.

Los resultados parecen indicar que los padres o cuidadores informan menos que los propios menores, sobre todo en lo que respecta a problemas de carácter internalizante. También se podría interpretar que los adolescentes sobrevaloran los problemas que afectan su estado emocional. Sin embargo, estudios previos indican que la información aportada por cada informante no es intercambiable y que lo habitual es que se encuentren diferencias dependiendo de quién valore el comportamiento del niño o adolescente (Rubio-Stipec, Fitzmaurice, Murphy \& Walker, 2003). Cada informante aporta datos valiosos que mejoran la utilidad de la evaluación realizada.

En todo caso, los resultados de esta investigación indican que las informaciones de padres y cuidadores correlacionaron de manera similar con las aportadas por los propios adolescentes, con independencia del grupo al que pertenecían. Es más, los cuidadores mostraron mayor acuerdo con los informes de los participantes del grupo institucionalizado, lo que no permite atribuir a su desconocimiento de los menores los diferentes resultados que arrojan ambos tipos de informantes, por separado, a lo largo del estudio.

Los hallazgos obtenidos permiten señalar que los niños y adolescentes institucionalizados informan 
de más problemas de comportamiento que aquellos que viven con sus familias. Pese a que a juicio de los adultos no existen diferencias entre ambos grupos, los propios menores institucionalizados sí informan de una mayor problemática psicológica que sus compañeros no institucionalizados. Dado que ambos tipos de informantes ofrecen visiones diferentes de la realidad psicológica, este dato no debe ser desdeñado si quiere mejorarse la atención que se presta a este colectivo.

Por otra parte, aunque los niños, niñas y adolescentes institucionalizados pueden percibir una problemática mayor que la que informan los no institucionalizados, estas diferencias no son tan relevantes como para que padres y/o cuidadores las detecten. Parece evidente que, como ya señalaban Armsdem et al. (2000), muchos menores institucionalizados funcionan normalmente.

Una de las principales limitaciones del estudio está en el hecho de la naturaleza transversal de la investigación, por lo tanto, los resultados no deben ser interpretados en términos evolutivos. Tampoco se puede saber si las diferencias encontradas pueden ser atribuidas a la institucionalización en centros de bienestar infantil o a las condiciones previas a la institucionalización vividas por los propios menores (orfandad, abandono, maltrato...). Además, por la propia naturaleza de las instituciones y las dificultades derivadas de las normativas de protección, resultó imposible acceder a datos individuales sobre la permanencia de los niños en las instituciones en las que estaban acogidos, sus situaciones particulares y de vida previas a su ingreso (p. ej., nivel socioeconómico o educativo de sus padres o familias de origen). En futuros estudios deberá considerarse el peso o importancia que las características específicas de los niños, cuidadores y centros de protección pueden tener en relación con la problemática psicológica detectada. Finalmente, la falta de puntos de corte para población venezolana impide detectar, con los instrumentos utilizados, la existencia o no de trastornos o síndromes clínicos, y ofrecer la prevalencia de los mismos, de forma que pueda ser analizada en relación con otras poblaciones.

La escasez de datos procedentes de muestras latinas, en relación con niños y adolescentes ins- titucionalizados impide contar con un punto de referencia sobre este tipo de población y poder así contrastar resultados y hacer comparaciones adecuadas. Precisamente, por esto son necesarios más estudios realizados con niños y adolescentes institucionalizados en Venezuela y otros países de habla hispana, que consideren diferentes etapas de su desarrollo evolutivo, que involucren a múltiples informantes y que otorguen una visión más amplia sobre el tema.

Este estudio evidencia que los niños y adolescentes institucionalizados en centros de bienestar infantil o las entidades de atención (acogimiento residencial) en Venezuela, presentan más problemas de comportamiento de todo tipo (internalizado, externalizado y mixto) que los encontrados en la población general. También muestran una peor situación académica y menores competencias psicosociales. Con independencia de que los problemas detectados sean consecuencia de la propia institucionalización, o resultado de las condiciones que llevaron a su acogimiento residencial, deberían recibir una mayor atención por parte de las entidades y autoridades responsables.

\section{Referencias}

Abad, J., Forns, M., Amador, J. \& Martorell, B. (2000). Fiabilidad y validez del Youth Self-Report en una muestra de adolescentes. Psicothema, 12(1), 49-54.

Achenbach, T., Becker, A., Döpfner, M., Heiervang, E., Roessner, V., Steinhausen, H., et al. (2008). Multicultural assessment of child and adolescent psychopathology with ASEBA and SDQ instruments: Research findings, applications, and future directions. Journal of Child Psychology and Psychiatry, 49(3), 251-275.

Achenbach, T. \& Edelbrock, C. (1983). Manual for the Child Behavior Checklist and revised child behavior profile. Burlington, VT: Thomas. A. Achenbach.

Achenbach, T. M. \& Rescorla, L. A. (2001). Manual for ASEBA School-Age Forms $\mathcal{E}$ Profiles. Burlington, VT: University of Vermont.

Adoption and Foster Care Analysis and Reporting System. (2008). The AFCARS Report. Preliminary FY 2006 Estimates as of January 2008 (AFCARS Re- 
port No. 14). Washington, DC: U.S. Department of Health \& Human Services, Administration for Children \& families. Disponible en http://www. acf.hhs.gov/programs/cb/stats_research/afcars/tar/ report14.pdf

Armsden, G., Pecora, P., Payne, V. \& Szatkiewicz, J. (2000). Children placed in long-term foster care: An intake profile using the Child Behavior Checklist/4-18. Journal of Emotional and Behavioral Disorders, 8(1), 49-64.

Behen, M., Helder, E., Rothermel, R., Solomon, K. \& Chugani, H. (2008). Incidence of specific absolute neurocognitive impairment in globally intact children with histories of early severe deprivation. Child Neuropsychology, 14(5), 453-469.

Benavides, J. \& Miranda, S. (2007). Actitud crítica hacia el castigo físico en niños víctimas de maltrato infantil. Universitas Psychologica, 6(2), 309-318.

Betancourt, D. \& Andrade, P. (2008). La influencia del temperamento en problemas internalizados y externalizados en niños. Revista Intercontinental de Psicología y Educación, 10(1), 29-48.

Caprara, G. \& Pastorelli, C. (1989). Toward a reorientation of research on aggression. European Journal of Personality, 3(2), 121-138.

Doyle, R., Mick, E. \& Biederman, J. (2007). Convergence between the Achenbach Youth Self-Report and structured diagnostic interview diagnoses in ADHD and non-ADHD Youth. The Journal of Nervous and Mental Disease, 195(4), 350-352.

Dozier, M. \& Rutter, M. (2008). Challenges to the development of attachment relationships faced by young children in foster and adoptive care. En J. Cassidy \& P. Shaver (Eds.), Handbook of attachment: Theory, research, and clinical applications (2a ed., pp. 698-717). New York: Guilford.

Eapen, D. J. (2009). Institutionalized children: The underprivileged. International Journal of Nursing Practice, 15(5), 349-352.

Fondo de las Naciones Unidas para la Infancia. (2009). Progreso para la infancia: un balance sobre la protección de la niñez. Nueva York: Autor.

Fondo de las Naciones Unidas para la Infancia. Venezuela. (2012). Información del país. Recuperado el 15 de abril de 2012, de http://www.unicef.org/ venezuela/spanish/overview.html
Ghera, M., Marshall, P., Fox, N., Zeanah, C., Nelson, C., Smyke, A., et al. (2009). The effects of foster care intervention on socially deprived institutionalized children's attention and positive affect: Results from the BEIP study. Journal of Child Psychology and Psychiatry, 50(3), 246-253.

Gómez, E., Muñoz, M. \& Haz, A. (2007). Familias multiproblemáticas y en riesgo social: características e intervención. Psykhe, 16(2), 43-54.

Gómez, E., Muñoz, M. \& Santelices, M. (2008). Efectividad de las intervenciones en apego con infancia vulnerada y en riesgo social: un desafío prioritario para Chile. Terapia Psicológica, 26(2), 241-251.

Gribble, K. (2007). A model for caregiving of adopted children after institutionalization. Journal of Child and Adolescent Psychiatric Nursing, 20(1), 14-26.

Hetlinger, C., Simpkins, C. \& Combs, T. (2000). Using the CBCL to determine the clinical status of children in state custody. Children and Youth Services Review, 22(1), 55-73.

Hudziak, J., Copeland, W., Stanger, C. \& Wadsworth, M. (2004). Screening for DSM-IV externalizing disorders with the Child Behavior Checklist: A receiver-operating characteristic analysis. Journal of Child Psychology and Psychiatry, 45(7), 1299-1307.

Ison, M. \& Morelato, G. (2008). Habilidades sociocognitivas en niños con conductas disruptivas y víctimas de maltrato. Universitas Psychologica, 7(2), 357-367.

Johnson, R., Browne, K. \& Hamilton, C. (2006). Young children in institutional care at risk of harm. Trauma, Violence Eु Abuse, 7(1), 34-60.

Kreppner, J., O'Connor, T., Rutter, M. \& The English and Romanian Adoptees Study Team. (2001). Can inattention/overactivity be an institutional deprivation syndrome? Journal of Abnormal Child Psychology, 29(6), 513-528.

Lieberknecht, J. \& Dalbosco, D. (2007). Sintomas depressivos no contexto de institucionalização. Revista de Psiquiatría do Rio Grande do Sul, 29(3), 305-314.

Luoma, I., Kaukonen, P., Mäntymaa, M., Puura, K., Tamminen, T. \& Samelin, R. (2004). A longitudinal study of maternal depressive symptoms, negative expectations and perceptions of child 
problems. Child Psychiatry and Human Development, 35(1), 37- 53.

Mash, E. J. \& Graham, S. A. (2001). Clasificación y tratamiento de la psicopatología infantil. En V. Caballo \& M. A. Simón (Eds.), Manual de psicología clínica infantil y del adolescente. Trastornos generales (pp. 29-56). Madrid: Pirámide.

Méndez, L. \& González, L. (2002). Descripción de patrones de apego en menores institucionalizados con problemas conductuales. Revista de Psicología de la Universidad de Chile, 11(2), 75-92.

Montiel, C., Montiel, I. \& Peña, J. (2007). Datos normativos del Cuestionario sobre el Comportamiento de Niños-Versión para padres (CBCL) y versión para profesores (TRF), en una muestra de niños venezolanos. Psicología Conductual. Revista Internacional de Psicología Clínica y de la Salud, 15(1), 29-44.

Muñoz, M., Gómez, P. \& Santamaría, C. (2008). Pensamientos y sentimientos reportados por los niños ante la separación de sus padres. Universitas Psychologica, 7(2), 347-356.

Muris, P. \& Maas, A. (2004). Strengths and difficulties as correlates of attachment style in institutionalized and non-institutionalized children with belowaverage intellectual abilities. Child Psychiatry and Human Development, 34(4), 317-329.

Reijneveld, S., Brugman, E., Verhulst, F. \& Verloove, P. (2005). Area deprivation and child psychosocial problems. A national cross-sectional study among school-aged children. Social Psychiatry and Psychiatric Epidemiology, 40(1), 18-23.

Rescorla, L. A., Achenbach, T. M., Ivanova, M. Y, Dumenci, L., Almqvist, F., Bilenberg, N., et al. (2007). Behavioral and emotional problems reported by parents of children ages 6 to 16 in 31 societies. Journal of Emotional and Behavioral Disorders, 15(3), 130-142.

Rubio-Stipec, M., Fitzmaurice, G., Murphy, J. \& Walker, A. (2003). The use of multiple informants in identifying the risk factors of depressive and disruptive disorders: Are they interchangeable? Social Psychiatry and Psychiatric Epidemiology, 38(2), 51 58.

Rutter, M. (2000). Children in substitute care: Some conceptual considerations and research implica- tions. Children and Youth Services Review, 22(9), 685-703.

Rutter, M., O'Connor, T. G. \& The English and Romanian Adoptees Study Team. (2004). Are there biological programming effects for psychological development? Findings from a study of Romanian adoptees. Developmental Psychology, 40(1), 81-94.

Sameroff, A. J. \& Fiese, B. H. (2000). Transactional regulation: The developmental ecology of early intervention. En J. P. Shonkoff \& S. J. Meisels (Eds.), Handbook of early childhood intervention (pp. 135-159). New York: Cambridge University Press. Schmid, M., Goldbeck, L., Nutzel, J. \& Fegert, J. M. (2008). Prevalence of mental disorders among adolescents in German youth welfare institutions. BMC. Child and Adolescent Psychiatry and Mental Health, 2(1), 1-8.

Simsek, Z., Erol, N., Öztop, D. \& Münir, K. (2007). Prevalence and predictors of emotional and behavioral problems reported by teachers among institutionally reared children and adolescents in Turkish orphanages compared with community controls. Children and Youth Services Review, 29(7), 883-899.

Smokowski, P., Buchanan, R. \& Bacallao, M. (2009). Acculturation and adjustment in Latino adolescents: How cultural risk factors and assets influence multiple domains of adolescent mental health. Journal of Primary Prevention, 30(3-4), 371-393.

Smokowski, P., Rose, R. \& Bacallao, M. (2009). Acculturation and aggression in Latino adolescents: Modeling longitudinal trajectories from the Latino acculturation and health project. Child Psychiatry and Human Development, 40(4), 589-608.

Smyke, A. T., Koga, S. F., Johnson, D. E., Fox, N. A., Marshall, P. J., Nelson, C. A., et al. (2007). The caregiving context in institution-reared and familyreared infants and toddlers in Romania. Journal of Child Psychology and Psychiatry, 48(2), 210-218.

Smyke, A. T., Zeanah, C. H., Fox, N. A., Nelson, C. A \& Guthrie, D. (2010). Placement in foster care enhances quality of attachment among young institutionalized children. Child Development, 81(1), 212-223.

Valencia, M. \& Andrade, P. (2005). Validez del Youth Self Report para problemas de conducta en niños 
mexicanos. International Journal of Clinical and Health Psychology, 5(3), 499-520.

Van den Dries, L., Juffer, F., H. van IJzendoorn, M. \& Bakermans, M. (2009). Fostering security? A meta-analysis of attachment in adopted children. Children and Youth Services Review, 31(3), 410-421.

Welsh, J., Viana, A., Petrill, S. \& Mathias, M. (2007). Interventions for internationally adopted children and families: A review of the literature. Child and Adolescent Social Work Journal, 24(3), 285-311.
Zeanah, C., Smyke, A., Koga, S. \& Carlson, E. (2005). Attachment in institutionalized and community children in Romania. Child Development, 76(5), 1015-1028.

Zubeidat, I., Fernández-Parra, A., Sierra, J. \& Salinas, J. (2007). Comorbilidad de la ansiedad social específica y generalizada en adolescentes españoles. Psicothema, 19(4), 654-660. 